\title{
THE STRETCHED WEIGHT LATTICES OF A WEYL GROUP
}

\author{
DANIEL R. FARKAS ${ }^{1}$
}

\begin{abstract}
Consider a finite group of automorphisms of a lattice. The nonnegative cone is a fundamental domain (for the action of the group on the lattice) if and only if the group is a Weyl group and the lattice is isomorphic, as a module, to one of the group's stretched weight lattices.
\end{abstract}

In a recent paper [2], I had occasion to quote the following theorem of Bourbaki.

THEOREM 1 [1, VI $\S 3.4$, TheOREM 1, p. 188]. Suppose that $W$ is a Weyl group and $\Lambda$ is its weight lattice. Then the algebra of invariants of the group algebra, $\mathbf{C}[\Lambda]^{W}$, is a polynomial ring.

The argument offered there used very little of the detailed structure of the weight lattice. Indeed, it proves a bit more. Consider an $n$-dimensional Weyl group $W$ and fix a base for its root system. Let $\omega_{1}, \ldots, \omega_{n}$ be the fundamental dominant weights.

DEFinition. Suppose $S$ is a Z-lattice lying between the root lattice and the weight lattice. If there are positive integers $d_{1}, \ldots, d_{n}$ such that $S$ is the integral span of $d_{1} \omega_{1}, \ldots, d_{n} \omega_{n}$ then $S$ is called a stretched weight lattice.

It is known [1, p. 167] that every abelian group lying between the root lattice and the weight lattice is stable under the action of $W$. Hence $S$ is a $W$-module. Bourbaki actually proves

THEOREM $1+$. If $S$ is a stretched weight lattice for the Weyl group $W$, then $\mathbf{C}[S]^{W}$ is a polynomial ring.

The point of this note is that the stretched weight lattices have a remarkable abstract characterization. Recall that if a group $G$ acts on a set $X$ then a fundamental domain for the action is a subset $D$ of $X$ such that

(i) for each $x \in X$ there is a $g \in G$ such that $g \cdot x \in D$,

(ii) $g \cdot d_{1}=d_{2}$ for $d_{1}, d_{2} \in D$ and some $g \in G$ implies $d_{1}=d_{2}$.

For instance, if $W$ is a Weyl group with fundamental dominant weights $\omega_{1}, \ldots, \omega_{n}$ as above, then $\Lambda^{+}=\left\{\sum_{i=1}^{n} a_{i} \omega_{i} \mid a_{i}\right.$ is a nonnegative integer for $\left.i=1, \ldots, n\right\}$ is a fundamental domain for the action of $W$ on the weight lattice $\Lambda$. It is easy to see, as a consequence, that when $S$ is a stretched weight lattice spanned by $d_{1} \omega_{1}, \ldots, d_{n} \omega_{n}$, then $\Lambda^{+} \cap S$ (the cone of nonnegative integer combinations of the $\left.d_{j} \omega_{j}\right)$ is a fundamental domain for the action of $W$ on $S$. In a very general sense, the converse is true.

Received by the editors November 8, 1983 and, in revised form January 13, 1984.

1980 Mathematics Subject Classification. Primary 20H15, 17 B20.

Key words and phrases. Weyl group, weight lattice, fundamental domain.

${ }^{1}$ Partially supported by an NSF grant. 
THEOREM 2. Let $M=\mathbf{Z}^{n}$ with standard basis $e_{1}, \ldots, e_{n}$. Assume that $G$ is a finite subgroup of $\mathrm{GL}(M)$ and $\left\{\sum_{i=1}^{n} a_{i} e_{i} \mid a_{i}\right.$ is a nonnegative integer $\}$ is a fundamental domain for the induced action on $M$. Then $G \subset \mathrm{GL}(n, \mathbf{R})$ is a Weyl group and, as a $G$-module, $M$ is isomorphic to a stretched weight lattice.

1. Examples. Assume that $W$ is a Weyl group with root system $R$. The weight lattice $\Lambda$ is clearly a stretched weight lattice. We shall indicate how the other stretched weight lattices can be obtained from it by doubling certain fundamental dominant weights.

Fix a base for the root system $R$. If $R$ decomposes as a direct sum of root systems $R_{1}$ and $R_{2}$ then $W=W_{1} \times W_{2}$ and $\Lambda=\Lambda_{1} \oplus \Lambda_{2}$ in a compatible way. Hence if $S$ is a stretched weight lattice for $W$, then $S=S_{1} \oplus S_{2}$, where each $S_{i}$ is a stretched weight lattice for $W_{i}$. It follows that any stretched weight lattice is a direct sum of stretched weight lattices for irreducible Weyl groups.

The next proof is left to the reader.

Lemma 3. Assume that $D$ and $C$ are integer $n \times n$ matrices. Then $D \cdot \mathbf{Z}^{n} \supset$ $C \cdot \mathbf{Z}^{n}$ if and only if there is an integer matrix $E$ such that $D E=C$.

If $\Lambda$ is the weight lattice for $W$ with distinguished basis the fundamental dominant weights, then the root lattice is $C \cdot \Lambda$, where $C$ is the transpose of the Cartan matrix for the root system. Recall that a stretched weight lattice for $W$ has the form $D \cdot \Lambda$, where $D$ is a diagonal matrix

$$
\left[\begin{array}{llll}
d_{1} & & & \\
& d_{2} & & \\
& & \ddots & \\
& & & d_{n}
\end{array}\right] .
$$

Moreover, any stretched weight lattice contains the root lattice. Thus $D \cdot \Lambda \supset C \cdot \Lambda$. By Lemma 3, there is an integer matrix $E$ with $D E=C$. That is, $d_{j}$ divides every entry in the $j$ th column of the Cartan matrix.

Now inspect the Cartan matrices for the irreducible root systems [1, pp. 250 275]. For systems of any type except $A_{1}, C_{2}$ and $B_{l}(l \geq 2)$, every column of the Cartan matrix has greatest common divisor 1. In all of these cases, the only stretched weight lattice is the weight lattice itself. The remaining root systems have a Cartan matrix in which all columns but one are unimodular and the remaining column has greatest common divisor 2. For each of these systems, one may double a single fundamental dominant weight to obtain a "new" stretched weight lattice. Notice that for the root systems we listed, weight lattice/root lattice is the group of order two. Hence the stretched weight lattice with the doubled weight is isomorphic to the corresponding root lattice.

It remains conceivable that the root and weight lattices we have singled out are heavily disguised isomorphic copies of each other. The root and weight lattices for $A_{1}$ are clearly isomorphic. While this is not the case for $B_{2}$, there is an automorphism $\theta$ of the Weyl group (the dihedral group of order 8) and a Z-module isomorphism

$$
\Psi: \text { weight lattice } \rightarrow \text { root lattice }
$$


such that $\Psi(g \cdot a)=\left({ }^{\theta} g\right) \cdot \Psi(a)$ for all $g$ in the group. However, when $l \geq 3$ the root lattice for $B_{l}$ is not isomorphic to the weight lattice, even in this twisted sense.

To outline a proof, recall that the root lattice for $B_{l}$ is the free $\mathbf{Z}$-module on $\varepsilon_{1}, \ldots, \varepsilon_{l}$ and the Weyl group is $W=(\mathbf{Z} / 2 \mathbf{Z})^{l} S_{l}$. Here an element of $\mathbf{Z} / 2 \mathbf{Z}$ is identified with \pm 1 and $\left(a_{1}, \ldots, a_{l}\right)$ acts on $\varepsilon_{i}$ by sending it to $a_{i} \varepsilon_{i}$; an element of the symmetric group $S_{l}$ acts on $\left\{\varepsilon_{1}, \ldots, \varepsilon_{l}\right\}$ by permuting the indices in the obvious way. In summary, the root lattice has a set of $2 l$ members $\left\{ \pm \varepsilon_{i} \mid i=1, \ldots, l\right\}$ which spans it and is stabilized by the Weyl group. Any $W$-lattice twist-isomorphic to the root lattice must also have such a stable spanning set.

We claim that if $l \geq 3$ and if $V=\left\{ \pm v_{i} \mid i=1, \ldots, l\right\}$ is any $W$-stable set of $2 l$ nonzero vectors in the real span of the root lattice, then $V=\left\{ \pm \lambda \varepsilon_{i}\right\}$ for some $\lambda \neq 0$. Indeed, suppose that $v_{1}=\sum_{j=1}^{l} \lambda_{j} \varepsilon_{j}$ with exactly $t$ of the coefficients nonzero. If $t=1$ then $v_{1}=\lambda_{r} \varepsilon_{r}$ for some $r$; it follows easily that $V=\left\{ \pm \lambda_{r} \varepsilon_{i} \mid i=1, \ldots, l\right\}$, whence the claim. If $t=l$ then the action of $(\mathbf{Z} / 2 \mathbf{Z})^{l}$ on $v_{1}$ produces $2^{l}$ distinct vectors. But $2^{l}>2 l$, producing too many vectors in $V$. So assume $1<t<l$ and say $\lambda_{1} \neq 0$. There are $\left(\begin{array}{c}l-1 \\ t-1\end{array}\right)$ subsets of $\varepsilon_{2}, \ldots, \varepsilon_{l}$ with $t-1$ elements and the stabilizer of 1 in $S_{l}$ acts transitively on them. Thus the action of $S_{l}$ on $v_{1}$ produces at least $\left(\begin{array}{c}l-1 \\ t-1\end{array}\right)$ vectors in $V$ of the form $\lambda_{1} \varepsilon_{1}+\cdots$ and with "distinct supports". If we move these vectors by $(\mathbf{Z} / 2 \mathbf{Z})^{l}$ we obtain at least $2^{t}\left(\begin{array}{c}l-1 \\ t-1\end{array}\right) \geq 4(l-1)>2 l$ distinct elements in $V$.

The weight lattice for $B_{l}$ is the integral span of $\varepsilon_{1} \cdots \varepsilon_{l-1}$ and $s=\frac{1}{2}\left(\varepsilon_{1}+\cdots+\varepsilon_{l}\right)$. It obviously has no spanning set of the form $\left\{ \pm \lambda \varepsilon_{i} \mid i=1, \ldots, l\right\}$.

One additional comment should be made. Suppose that $W$ is an arbitrary Weyl group and $\Lambda$ is its weight lattice. Any $W$-lattice twist-isomorphic to $\Lambda$ has the same fundamental domains as $\Lambda$. As a corollary to Theorem 2 , we conclude that a "twisted" weight lattice is isomorphic to some stretched weight lattice.

2. Weyl groups. We prove Theorem 2 in a large number of small steps. The lattice $M=\mathbf{Z}^{n}$ has the standard basis $e_{1}, \ldots, e_{n}$. We regard $M$ as imbedded in $\mathbf{R}^{n}$ using this basis. Let $P$ be the closed positive quadrant

$$
P=\left\{\left(x_{1}, \ldots, x_{n}\right) \in \mathbf{R}^{n} \mid x_{i} \geq 0 \text { for } i=1, \ldots, n\right\} .
$$

The basic assumption is that $G \subset \mathrm{GL}(M)$ is a finite group and $P \cap \mathbf{Z}^{n}$ is a fundamental domain for its action on $M=\mathbf{Z}^{n}$. First observe that $P \cap \mathbf{Q}^{n}$ is a fundamental domain for the action of $G$ on $\mathbf{Q}^{n}$; this follows from the corresponding assumption about $\mathbf{Z}^{n}$ by "clearing denominators". Second, one can average the standard inner product on $\mathbf{R}^{n}$ over $G$ to obtain a new inner product that is "G-invariant". Equivalently, $G$ lies in the orthogonal group determined by the new inner product. We use the $G$-invariant norm in constructing all of the balls below.

Lemma 4. Suppose $x \in \operatorname{int} P$ and $g \in G$. Then $g(x)=x$ implies $g=1$.

Proof. Let $B$ be an open ball with center $x$ contained in int $P$. Then $g(B)=B$ because $g$ is orthogonal. If $y$ is a vector with rational coordinates in $B$, then $g(y)=y$ by (ii) in the definition of fundamental domain. But the rational vectors in $B$ lie on infinitely many lines through the origin. Thus $g=1$.

LEMMA 5. If $x$ lies on the boundary of $P$ then there is a $g \neq 1$ in $G$ such that $g(x)=x$. 
PROOF. First suppose that $y$ is a rational vector on the boundary. Choose a sequence $y_{m} \rightarrow y$ with $y_{m} \in P$. For each $y_{m}$ there is a $g_{m} \neq 1$ in $G$ with $g_{m}\left(y_{m}\right) \in P$. Since $G$ is finite, we can drop to a subsequence and assume that $g\left(y_{m}\right) \in P$ for a single $g \neq 1$ in $G$. Now $P$ is closed: $g\left(y_{m}\right) \rightarrow g(y)$, where $g(y) \in P \cap \mathbf{Q}^{n}$. Thus $g(y)=y$ (property (ii) of fundamental domains).

Another continuity-and-subsequence argument allows us to get from a rational vector to an arbitrary one.

By an axis hyperplane we shall mean one of the

$$
\mathscr{H}_{i}=\left\{\left(x_{1}, \ldots, x_{n}\right) \in \mathbf{R}^{n} \mid x_{i}=0\right\} .
$$

We call $P \cap \mathscr{H}_{i}$ a face of $P$.

LEMMA 6. If $\sigma_{i}$ is orthogonal reflection in the axis hyperplane $\nvdash_{i}$, then $\sigma_{i} \in G$.

PROOF. Let $\mathcal{F}_{i}$ be the $i$ th face of $P$. According to Lemma 5,

$$
\mathcal{F}_{i} \subset \bigcup_{g \neq 1}\left\{x \in \mathbf{R}^{n} \mid(g-1)(x)=0\right\},
$$

a finite union of subspaces. A standard argument implies that there is a $g \neq 1$ with $\mathcal{F}_{i} \subset\left\{x \in \mathbf{R}^{n} \mid g(x)=x\right\}$. Thus some $g \neq 1$ in $G$ fixes each point in $\mathscr{H}_{i}$. Since $g$ is orthogonal, it must be a reflection.

Let $W$ denote the group generated by the orthogonal reflections $\sigma_{1}, \ldots, \sigma_{n}$. According to Lemma 6 , we have $W \subset G$, whence $W$ is finite.

LEMMA 7. $P$ is a fundamental domain for the action of $W$ on $\mathbf{R}^{n}$.

PROOF. The lemma is a corollary to $[\mathbf{1}, \mathrm{V} \S 3.3, \mathrm{p} .75]$ once we show that if $\mathcal{H}_{i}$ is an axis hyperplane and $g \in W$, then $g\left(\mathcal{H}_{i}\right) \cap P$ is a face.

Suppose $x \in \mathscr{H}_{i}$. Then $\left(g \sigma_{i} g^{-1}\right)(g x)=g x$. By Lemma 4, no interior points of $P$ can be fixed points. Thus $g\left(\mathscr{H}_{i}\right) \cap P$ lies on the boundary of $P$.

LEMMA 8. $G=W$.

ProOF. Pick any $x$ in $\mathbf{Q}^{n} \cap$ int $P$. If $g \in G$, then Lemma 7 implies that there is an $h \in W$ such that $h(g(x)) \in P$. Thus $h g(x)=x$. By Lemma $4, h g=1$. We conclude that $g=h^{-1} \in W$.

LEMMA 9. G is a Weyl group.

PROOF. $W$ has all of the properties required to make it a Weyl group, as listed in $\{1$, Proposition 9, VI $\S 2.5]$, with the possible exception of being effective. Recall that $W$ is effective if 0 is the only simultaneous fixed point for all members of $W$.

So assume that $x \in \mathbf{R}^{n}$ is such that $g(x)=x$ for all $g \in G$. According to Lemma 7 , we must have $x \in P$. Similarly, $-x \in P$. This happens only when $x=0$.

LEMMA 10. $M$ is isomorphic to a $G$-module which lies between the root lattice and the weight lattice for $G$.

PROOF. Given any lattice on which a finite reflection group acts effectively, one can find a compatible root system inside the lattice so that the lattice is positioned as required in the lemma. More details can be found in Theorem 9 of [2].

Assume now that a base for the root system of $G$ is chosen and $M$ lies between the root and weight lattices. Let $e_{1}=(1,0, \ldots, 0), \ldots, e_{n}=(0, \ldots, 0,1)$ be the 
distinguished basis for $M$ and let $\omega_{1}, \ldots, \omega_{n}$ be the fundamental dominant weights a basis for the weight lattice $\Lambda$. Lemmas 7 and 8 state that $P$ is the closure of a Weyl chamber for $G$. The collection of dominant weights (the nonnegative linear combinations of $\left.\omega_{1}, \ldots, \omega_{n}\right)$ also comprise the closure of a Weyl chamber. Thus there is a $g \in G$ which carries $P$ bijectively to the cone of dominant weights. Therefore we may assume that $e_{1}, \ldots, e_{n}$ lie in this cone.

Proof OF TheOrem 2. The basis sets $\left\{e_{1}, \ldots, e_{n}\right\}$ and $\left\{\omega_{1}, \ldots, \omega_{n}\right\}$ have the following remarkable property: each member of one set can be written as a nonnegative linear combination of members in the other. Moreover, each $e_{j}$ is a nonnegative integer combination of the $\omega_{1}, \ldots, \omega_{n}$. It follows that if $A$ is the change of basis matrix from $\left\{e_{1}, \ldots, e_{n}\right\}$ to $\left\{\omega_{1}, \ldots, \omega_{n}\right\}$, then $A$ has nonnegative integer entries and $A^{-1}$ has nonnegative real entries.

Set $A=\left(a_{i j}\right)$ and $A^{-1}=\left(b_{i j}\right)$. For each $i$ there exists at least one $\pi(i)$ such that $a_{i \pi(i)} b_{\pi(i) i} \neq 0$. If $a_{j \pi(i)} \neq 0$ for $j \neq i$, then $a_{j \pi(i)} b_{\pi(i) i}>0$, forcing the $(j, i)$-entry of $A \cdot A^{-1}$ to be strictly positive. Hence $a_{j \pi(i)}=0$ for all $j \neq i$. In other words, each row of $A$ has exactly one nonzero entry and the same is true for each column.

We conclude that there are positive integers $d_{1}, \ldots, d_{n}$ and a permutation $\pi \in S_{n}$ such that $e_{j}=d_{j} \omega_{\pi(j)}$.

NOTE ADDED IN PROOF. I am indebted to Robert Steinberg for pointing out to me that the root lattice for a root system of type $B_{n}$ is isomorphic, as a module over its Weyl group, to the weight lattice for type $C_{n}$. Consequently, the distinction between stretched weight lattice and ordinary weight lattice only exists once a root system has been specified. Theorem 2 would be better stated as follows: the nonnegative cone is a fundamental domain for a finite group $G$ of automorphisms of $\mathbf{Z}^{n}$ if and only if $G$ is a reflection group and, for some choice of root system, it becomes a Weyl group with $\mathbf{Z}^{n}$ as weight lattice.

\section{REFERENCES}

1. N. Bourbaki, Groupes et algèbres de Lie. IV, V, VI, Hermann, Paris, 1968.

2. D. R. Farkas, Multiplicative invariants, Enseign. Math. (to appear).

Department of Mathematics, Virginia Polytechnic Institute and State UNIVERSITY, BLACKSBURG, VIRGINIA 24061 